\title{
Transmission enhancement in loss-gain multilayers by resonant suppression of reflection
}

Novitsky, Denis V.; Tuz, Vladimir R.; Prosvirnin, Sergey L.; Lavrinenko, Andrei V.; Novitsky, Andrey V.

Published in:

Physical Review B (Condensed Matter and Materials Physics)

Link to article, DOI:

10.1103/PhysRevB.96.235129

Publication date:

2017

Document Version

Publisher's PDF, also known as Version of record

Link back to DTU Orbit

Citation (APA):

Novitsky, D. V., Tuz, V. R., Prosvirnin, S. L., Lavrinenko, A. V., \& Novitsky, A. V. (2017). Transmission enhancement in loss-gain multilayers by resonant suppression of reflection. Physical Review $B$ (Condensed Matter and Materials Physics), 96(23), [235129]. https://doi.org/10.1103/PhysRevB.96.235129

\section{General rights}

Copyright and moral rights for the publications made accessible in the public portal are retained by the authors and/or other copyright owners and it is a condition of accessing publications that users recognise and abide by the legal requirements associated with these rights.

- Users may download and print one copy of any publication from the public portal for the purpose of private study or research.

- You may not further distribute the material or use it for any profit-making activity or commercial gain

- You may freely distribute the URL identifying the publication in the public portal 


\title{
Transmission enhancement in loss-gain multilayers by resonant suppression of reflection
}

\author{
Denis V. Novitsky, ${ }^{1,2, *}$ Vladimir R. Tuz, ${ }^{3,4}$ Sergey L. Prosvirnin, ${ }^{4,5}$ Andrei V. Lavrinenko, ${ }^{6}$ and Andrey V. Novitsky ${ }^{6,7, \dagger}$ \\ ${ }^{1}$ B. I. Stepanov Institute of Physics, National Academy of Sciences of Belarus, 68 Nezavisimosti Avenue, Minsk 220072, Belarus \\ ${ }^{2}$ ITMO University, 49 Kronverksky Pr., St. Petersburg 197101, Russia \\ ${ }^{3}$ International Center of Future Science, State Key Laboratory on Integrated Optoelectronics, College of Electronic Science and Engineering, \\ Jilin University, 2699 Qianjin Str., Changchun 130012, China \\ ${ }^{4}$ Institute of Radio Astronomy of National Academy of Sciences of Ukraine, 4 Mystetstv Street, Kharkiv 61002, Ukraine \\ ${ }^{5}$ School of Radio Physics, V.N. Karazin Kharkiv National University, 4 Svobody sq., Kharkiv 61022, Ukraine \\ ${ }^{6}$ DTU Fotonik, Technical University of Denmark, Ørsteds Plads 343, DK-2800 Kongens Lyngby, Denmark \\ ${ }^{7}$ Department of Theoretical Physics and Astrophysics, Belarusian State University, 4 Nezavisimosti Avenue, Minsk 220030, Belarus
}

(Received 11 September 2017; revised manuscript received 27 November 2017; published 19 December 2017)

\begin{abstract}
Using the transfer-matrix approach and solving time-domain differential equations, we analyze the loss compensation mechanism in multilayer systems composed of an absorbing transparent conductive oxide and dielectric doped with an active material. We reveal also another regime with the possibility of enhanced transmission with suppressed reflection originating from the resonant properties of the multilayers. For obliquely incident and evanescent waves, such enhanced transmission under suppressed reflection turns into the reflectionless regime, which is similar to that observed in the $\mathcal{P} \mathcal{T}$-symmetric structures, but does not require $\mathcal{P} \mathcal{T}$ symmetry. We infer that the reflectionless transmission is due to the full loss compensation at the resonant wavelengths of the multilayers.
\end{abstract}

DOI: 10.1103/PhysRevB.96.235129

\section{INTRODUCTION}

Metals play a tremendous role in modern optics and nanophotonics due to their ability for light concentration in tiny volumes [1]. Field enhancement is realized through excitation of propagating and/or localized surface plasmonsand can be used for strengthening linear and nonlinear lightmatter interactions [2,3]. However, electromagnetic waves in structures containing metals suffer from losses, and the beneficial plasmonic properties may fade away. One of the simplest transmission systems is a one-dimensional (1D) metal-dielectric multilayer structure. It attracts much attention nowadays, since it provides one of the possible implementations of so-called hyperbolic metamaterials (HMMs) [4,5]. HMMs are able to support waves with high wave numbers, which are typically evanescent in homogeneous dielectrics, thus allowing superresolution, the Purcell factor enhancement, and so on [6-8]. However, attenuation of transmitted waves greatly degrades the performance of the HMM-based devices.

Transparent conductive oxides (TCOs), such as tin-doped indium-oxide (ITO), aluminum-doped zinc-oxide (AZO), and gallium-doped zinc-oxide (GZO), exhibit reasonably low losses and, thus, can be employed as alternative plasmonic materials [9-13]. Another simple recipe for mitigating the absorption problem is loss compensation using amplifying materials. Optical gain can be delivered by low-dimensional electronic systems such as quantum dots and quantum wells, active impurities, or new phases in the semiconductor lattice, as well as by bulk semiconductors themselves [14]. In the case of atomically thin transition metal dichalcogenides the optical gain can be enhanced using the nanostructured plasmonic substrate as demonstrated in Ref. [15]. Using active slabs,

\footnotetext{
*dvnovitsky@gmail.com

†anov@fotonik.dtu.dk
}

losses can be compensated in surface plasmonic waveguides [16-18] and negative-refractive-index metamaterials [19,20] under optical and electrical pumping. Loss compensation in metal-dielectric multilayers is mainly directed towards the hyperbolic metamaterials improvement [21-23] and can be used for enhancement of spontaneous emission [24] ameliorating of surface plasmon resonances [25], as well as for tuning gain/absorption spectra in graphene HMMs [26].

The losses in coalescence with gain lead to the remarkable property of the multilayers known as the parity-time $(\mathcal{P} \mathcal{T})$ symmetry. Optical $\mathcal{P} \mathcal{T}$ symmetry is transferred from quantum mechanics of non-Hermitian Hamiltonians [27]. It can be realized by the sequencing of active and passive structural components, which for the complex 1D permittivity reads $\varepsilon(z)=\varepsilon^{*}(-z)$ (here ${ }^{*}$ stands for the complex conjugate). $\mathcal{P} \mathcal{T}$ symmetry opens new opportunities for light control $[28,29]$ due to nonreciprocity of light propagation [30], anisotropic transmission resonances [31], and unidirectional invisibility [32]. It influences light localization in disordered structures [33] and manifests new regularities of optical switching and soliton generation in nonlinear systems $[34,35]$.

In most of the theoretical works gain in multilayers is modeled phenomenologically through the imaginary part of the complex permittivity. However, realistic modeling should include the temporal dynamics of light interaction with the amplifying medium. Only within the dynamical framework one can describe the fast processes and influence of the inhomogeneous field distribution inside a gain slab, as well as correctly predict establishment of the stationary state or lasing in metal-dielectric structures [36-38]. For the multilayer we reveal the frames of applicability of the transfer-matrix method using comparison with the inhouse finite-difference time-domain (FDTD) method [39,40]. The FDTD approach describes the frequency-dispersive metallike material response with the time-dependent equations, while the Bloch equations are employed for dielectric having 
resonant gain. Here we combine the advantages of the loss suppression in TCOs (AZO) and amplification in a gain material (with, i.e., quantum dots).

The paper is organized as follows. In Sec. II, we discuss the main equations governing light propagation in metaldielectric multilayers. Section III delivers a full-temporal evidence of loss compensation and signal amplification in active systems with and without saturation. The effect of resonant low-reflection transmission is introduced in Sec. IV on the basis of calculations with both the FDTD and transfermatrix techniques. Development of the lasinglike behavior, limiting the applicability of the transfer-matrix method, is studied as well. The resonant reflectionless transmission is observed in the case of obliquely incident light and evanescent waves, but the electromagnetic states differ from those of the $\mathcal{P} \mathcal{T}$-symmetric systems as shown in Sec. V. Section VI summarizes the article.

\section{MODELING LOSS AND GAIN IN TIME DOMAIN}

We consider a periodic planar structure composed of alternating metal-like and dielectric layers (Fig. 1) and illuminated by normally incident monochromatic light [convention $\exp (-i \omega t)$ is assumed, where $\omega$ is the angular frequency]. The permittivity of the metal-like slabs can be written according to the Drude model as

$$
\varepsilon_{m}(\omega)=\varepsilon_{\infty}-\frac{\omega_{p}^{2}}{\omega^{2}+i \gamma \omega},
$$

where $\varepsilon_{\infty}$ is the high-frequency dielectric constant, $\omega_{p}$ is the plasma frequency, and $\gamma$ is the electron plasma damping. Since we describe light propagation in the metal-dielectric structure in the time domain, Eq. (1) should be converted into a differential equation with respect to time. Introducing derivative $\partial / \partial t$ instead of $-i \omega$, we straightforwardly arrive at the oscillation equation for polarization

$$
\frac{d^{2} P}{d t^{2}}+\gamma \frac{d P}{d t}=\omega_{p}^{2} E .
$$

From here on we assume that the wave is linearly polarized, and we can use the scalar quantities of electric field $E$ and polarization $P$. Then light dynamics in the metal-like material is governed by the wave equation

$$
\frac{\partial^{2} E}{\partial z^{2}}-\frac{\varepsilon_{\infty}}{c^{2}} \frac{\partial^{2} E}{\partial t^{2}}=\frac{1}{c^{2}} \frac{\partial^{2} P}{\partial t^{2}} .
$$

Having carrier frequency $\omega$, we represent polarization $P=p(t, z) \exp [-i(\omega t-k z)]$ and electric field

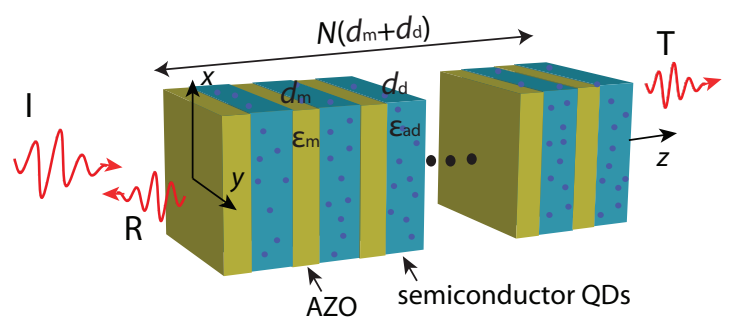

FIG. 1. Sketch of the system under investigation: $N$ periods of the alternating lossy AZO and amplifying dielectric slabs.
$E=A(t, z) \exp [-i(\omega t-k z)]$ in terms of the complex amplitudes $p(t, z)$ and $A(t, z)$. Then Eqs. (2) and (3) read as

$$
\begin{gathered}
\frac{d^{2} p}{d \tau^{2}}+\eta \frac{d p}{d \tau}+\zeta p=\alpha A, \\
\frac{\partial^{2} A}{\partial \xi^{2}}-\varepsilon_{\infty} \frac{\partial^{2} A}{\partial \tau^{2}}+2 i \frac{\partial A}{\partial \xi}+2 i \varepsilon_{\infty} \frac{\partial A}{\partial \tau}+A\left(\varepsilon_{\infty}-1\right) \\
=\frac{\partial^{2} p}{\partial \tau^{2}}-2 i \frac{\partial p}{\partial \tau}-p,
\end{gathered}
$$

where $\tau=\omega t$ and $\xi=k z$ are the dimensionless time and distance, $k=\omega / c$ is the vacuum wave number, $c$ is the speed of light in free space, $\eta=-2 i+\gamma / \omega, \zeta=-1-i \gamma / \omega$, and $\alpha=\omega_{p}^{2} / \omega^{2}$. Equations (4) and (5) can be numerically solved using the FDTD approach proposed earlier for the examination of nonlinear metamaterials [39].

The second component of the structure is an active dielectric (gain medium) modeled as a homogeneously broadened twolevel medium. Light propagates in this medium in concordance with the Maxwell-Bloch equations, that is, the system of differential equations for the dimensionless electric-field amplitude $\Omega=(\mu / \hbar \omega) A$ (normalized Rabi frequency), complex amplitude of the atomic polarization $\rho$, and difference between populations of ground and excited states $w$ [41]:

$$
\begin{gathered}
\frac{d \rho}{d \tau}=i l \Omega w+i \rho \delta-\gamma_{2} \rho, \\
\frac{d w}{d \tau}=2 i\left(l^{*} \Omega^{*} \rho-\rho^{*} l \Omega\right)-\gamma_{1}\left(w-w_{e q}\right), \\
\frac{\partial^{2} \Omega}{\partial \xi^{2}}-n_{d}^{2} \frac{\partial^{2} \Omega}{\partial \tau^{2}}+2 i \frac{\partial \Omega}{\partial \xi}+2 i n_{d}^{2} \frac{\partial \Omega}{\partial \tau}+\left(n_{d}^{2}-1\right) \Omega \\
=3 \epsilon l\left(\frac{\partial^{2} \rho}{\partial \tau^{2}}-2 i \frac{\partial \rho}{\partial \tau}-\rho\right),
\end{gathered}
$$

where $\mu$ is the dipole moment of the quantum transition, $\hbar$ is the reduced Planck constant, $\delta=\Delta \omega / \omega=\left(\omega_{0}-\omega\right) / \omega$ is the normalized frequency detuning, $\omega_{0}$ is the frequency of the atomic resonance, $\gamma_{1}=1 /\left(\omega T_{1}\right)$ and $\gamma_{2}=1 /\left(\omega T_{2}\right)$ are respectively normalized relaxation rates of population and polarization, $T_{1}\left(T_{2}\right)$ is the longitudinal (transverse) relaxation time, and $w_{e q}$ is the equilibrium population difference. Lightmatter coupling is described by the dimensionless parameter $\epsilon=\omega_{L} / \omega=4 \pi \mu^{2} C / 3 \hbar \omega$, where $\omega_{L}$ is the normalized Lorentz frequency and $C$ is the concentration (density) of two-level atoms. Quantity $l=\left(n_{d}^{2}+2\right) / 3$ is the so-called local-field enhancement factor originating from polarization of the background dielectric with refractive index $n_{d}$ by the embedded active (two-level) particles [42,43]. Further we numerically solve Eqs. (6)-(8) using essentially the same FDTD approach as in our previous publications (e.g., see [40]). It is worth noting that at the edges of the calculation region we apply perfectly matched layers (PMLs) and use the total field/scattered field (TF/SF) method to set the incident field. Solutions of both linear equations (4) and (5) in conductive layers and nonlinear equations (6)-(8) in dielectric layers yield the temporal evolution of light in the whole structure.

Equilibrium population difference $w_{e q}$ is the key parameter governing the gain properties of dielectric. Without loss of generality it can be considered as a parameter of the pumping 
intensity. Indeed, when there is no external pump, all two-level atoms are in the ground state, i.e., the difference between the populations of the ground and excited states is $w_{e q}=1$. In the opposite case of totally inverted medium, all atoms are excited by the pump and $w_{e q}=-1$. The medium is saturated, if both levels are populated equally in the state of equilibrium. This gainless case corresponds to $w_{e q}=0$ being equivalent to the absence of transitions between the levels; in other words, the absence of two-level atoms themselves. We are apparently interested in nonzero inversion, which can serve as a simple model of the gain medium.

At first, we will demonstrate the link between the equilibrium population difference and gain estimating the permittivity of the two-level medium in the steady-state approximation. Eliminating the time derivatives in Eqs. (6) and (7), we get the stationary microscopic polarization $\rho_{s t}$ and population difference $w_{s t}$ as follows:

$$
\begin{gathered}
\rho_{s t}=\frac{i \gamma_{2}-\delta}{\gamma_{2}^{2}+\delta^{2}} \frac{l w_{e q}}{1+|\Omega|^{2} / \Omega_{\mathrm{sat}}^{2}} \Omega, \\
w_{s t}=\frac{w_{e q}}{1+|\Omega|^{2} / \Omega_{\mathrm{sat}}^{2}},
\end{gathered}
$$

where $\Omega_{\text {sat }}=\sqrt{\gamma_{1}\left(\gamma_{2}^{2}+\delta^{2}\right) / 4 l^{2} \gamma_{2}}$ is the Rabi frequency corresponding to the saturation intensity. Such a medium with saturable nonlinearity is characterized by the effective permittivity

$$
\varepsilon_{a d}=n_{d}^{2}+4 \pi \mu C \rho_{s t} / E=n_{d}^{2}+\frac{K\left(-\delta+i \gamma_{2}\right)}{1+|\Omega|^{2} / \Omega_{\mathrm{sat}}^{2}},
$$

where $K=3 \omega_{L} l^{2} w_{e q} /\left[\omega\left(\gamma_{2}^{2}+\delta^{2}\right)\right]$. According to Ref. [42], additional enhancement of the resonant polarization is taken into account. Assuming that $n_{d}$ is real, the imaginary part of complex permittivity $\varepsilon_{a d}$ is a function of pumping parameter $w_{e q}$. In order to achieve a net gain, the pumping parameter should be obviously negative. Applying further simplifications of the exact resonance $\delta=0$ and low-intensity external radiation $|\Omega| \ll \Omega_{\text {sat }}$, Eq. (11) transforms to

$$
\varepsilon_{a d} \approx n_{d}^{2}+3 i l^{2} \omega_{L} T_{2} w_{e q} .
$$

This formula will be exploited further for calculations of the transmission and reflection spectra of the multilayers within the transfer-matrix method [44]. It is worth noting that according to Eq. (12) the imaginary addition to permittivity is influenced only by the transverse (coherence) relaxation time $T_{2}$. The longitudinal (population) relaxation time $T_{1}$ defines the saturation intensity that limits the validity range of Eq. (12).

In this paper, we use Al-doped $\mathrm{ZnO}$ (AZO) as a conducting material. According to optical characterization of AZO films [45], this material can be described by Eq. (1) with $\varepsilon_{\infty}=$ 3.45, $\omega_{p}=2 \pi \times 274.5 \mathrm{THz}$, and $\gamma=2 \pi \times 35 \mathrm{THz}$. As an active dielectric, we choose a semiconductor doped with quantum dots at the exact resonance having the following parameters [46,47]: $n_{d}=3.3, \omega_{L}=10^{11} \mathrm{~s}^{-1}, T_{1}=1 \mathrm{~ns}$, and $T_{2}=0.1 \mathrm{ps}$. Estimation of the permittivity imaginary part according to Eq. (12) yields $\left|\operatorname{Im} \varepsilon_{a d}\right| \lesssim 0.5$ at $w_{e q}=-1$. The corresponding gain coefficient $g=4 \pi \operatorname{Im}\left(\sqrt{\varepsilon_{a d}}\right) / \lambda \lesssim$ $5 \times 10^{3} \mathrm{~cm}^{-1}$ at wavelength $\lambda \sim 2 \mu \mathrm{m}$ is indeed attainable with current semiconductor technologies [48,49]. It is also worth noting that the choice of materials is not unique and the similar results could be obtained for other sets of materials, if geometry of the structure and light wavelengths are appropriately adjusted.

Hereinafter, normalized Rabi frequency $\Omega=\mu A / \hbar \omega$ is used instead of the electric field amplitude $A$. The values of $\Omega$ are written in the units of dimensionless relaxation rate $\gamma_{2}$. Throughout the text of the paper the initial value of the population difference is equal to the pumping parameter as $w(t=0)=w_{e q}$.

\section{ABSORPTION COMPENSATION IN LOSSY MULTILAYERS}

Let us apply the model described above to investigation of loss compensation in periodic planar metamaterials composed of alternating layers of AZO and dielectric with gain. Assuming that the thickness of the period is much less than light wavelength $\lambda$ and using the effective medium approach, we get the principle components of the effective medium permittivity tensor [7]

$$
\begin{gathered}
\varepsilon_{x x}=\varepsilon_{y y}=p \varepsilon_{m}(\omega)+(1-p) \varepsilon_{a d}, \\
\varepsilon_{z z}=\left(\frac{p}{\varepsilon_{m}(\omega)}+\frac{1-p}{\varepsilon_{a d}}\right)^{-1},
\end{gathered}
$$

where $p=d_{m} /\left(d_{m}+d_{d}\right)$ is the AZO filling factor (part of the multilayer volume occupied by AZO), and $d_{m}$ and $d_{d}$ are the thicknesses of the AZO and dielectric slabs, respectively. In Fig. 2 we show the real and imaginary parts of $\varepsilon_{x x}$ and $\varepsilon_{z z}$ for $p=1 / 3$ and $w_{e q}=0$ (i.e., $\varepsilon_{a d}=n_{d}^{2}$ ). The imaginary part of $\varepsilon_{a d}$ does not significantly affect the effective permittivities. In the wavelength range $\lambda \approx 2.2-3.1 \mu \mathrm{m}$ the real parts of the permittivity components have opposite signs: $\operatorname{Re} \varepsilon_{x x}>0$ and $\operatorname{Re} \varepsilon_{z z}<0$. This means that in this spectral range the multilayer can be considered as an HMM of type I (dielectric type) [7]. The similar effective permittivity was reported for the passive $\mathrm{AZO} / \mathrm{ZnO}$ multilayer [50]. In agreement with the KramersKronig relations, absorption grows within this wavelength range of the resonance as evidenced by the imaginary part of the permittivity in Fig. 2(b). Our goal is to compensate for absorption by embedding a gain medium into the multilayer.

We consider a subwavelength multilayer having $N=50$ periods. The thicknesses of the AZO and dielectric slabs are $d_{m}=0.1 \mu \mathrm{m}$ and $d_{d}=0.2 \mu \mathrm{m}$, respectively, i.e., the filling factor equals $p=1 / 3$, as in Fig. 2. We study temporal dynamics of a monochromatic continuous wave (cw) at $\lambda=$ $2.5 \mu \mathrm{m}$. Reflection $R$ and transmission $T$ coefficients depend on pumping parameter $w_{e q}$ for small incident amplitude $\Omega_{0}=10^{-4} \gamma_{2} \ll \Omega_{\text {sat }}$ as demonstrated in Figs. 3(a) and 3(b), respectively. In the gainless case, the stationary reflection level is about $37 \%$, while the transmission is less than $1 \%$. The rest energy of the incident wave is absorbed by the structure. Turning on gain in the dielectric slabs results in the gradual increase of both reflection and transmission up to 1.38 and 0.30 , respectively, at the maximum pumping level. The oscillations of reflected and transmitted signals seen at $w_{e q}=-1$ are due to the abrupt switching on of the incident wave and can be eliminated with a smoothly 

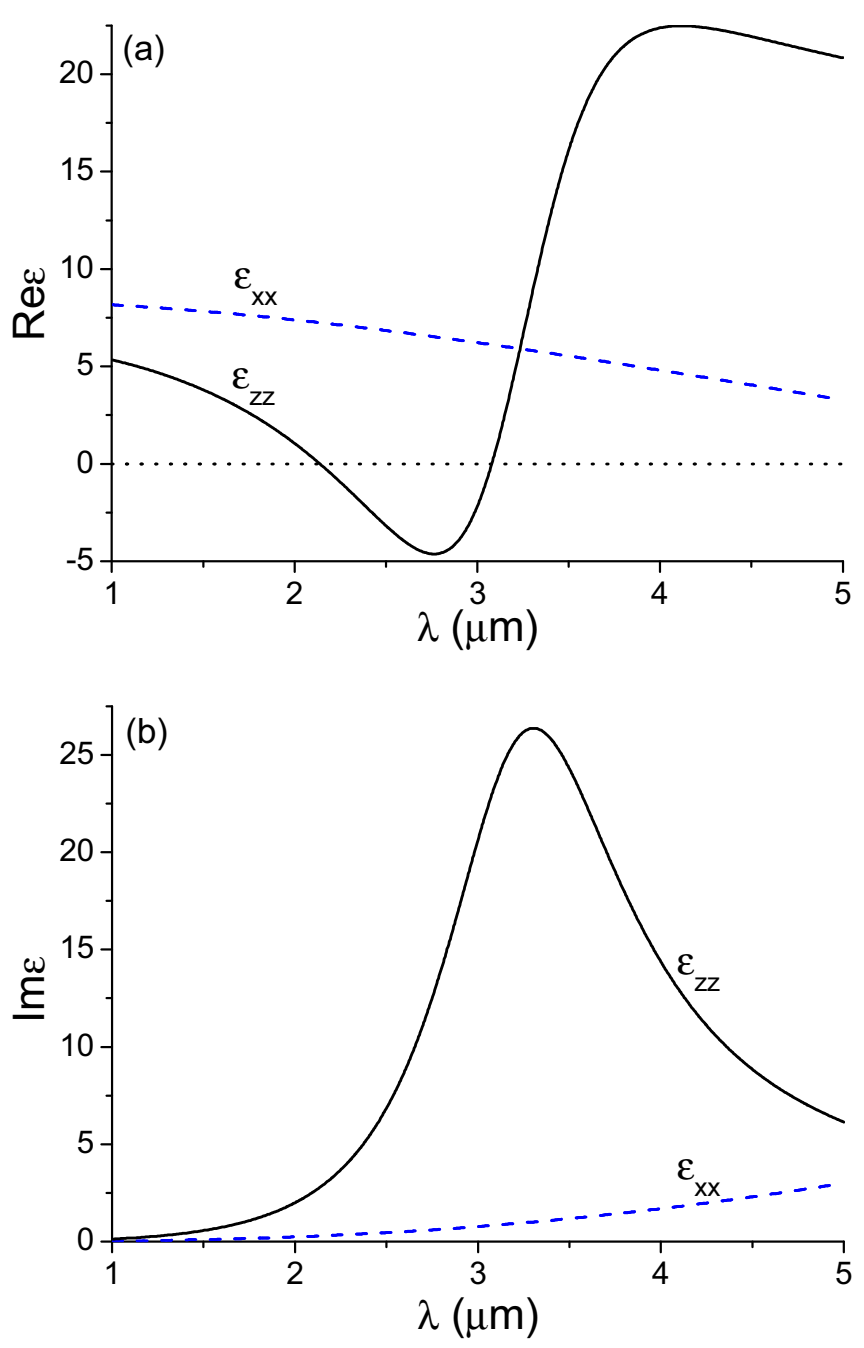

FIG. 2. (a) Real and (b) imaginary parts of the effective permittivity tensor components of the AZO-dielectric multilayer $(N=$ 50) structure. Parameters of the AZO slab: $\varepsilon_{\infty}=3.45, \omega_{p}=2 \pi \times$ $147.8 \mathrm{THz}, \gamma=2 \pi \times 35 \mathrm{THz}$, and $d_{m}=0.1 \mu \mathrm{m}$. Parameters of the gainless $\left(w_{e q}=0\right)$ dielectric slab: $n_{d}=3.3, \omega_{L}=10^{11} \mathrm{~s}^{-1}, T_{1}=1$ ns, $T_{2}=0.1 \mathrm{ps}$, and $d_{m}=0.2 \mu \mathrm{m}$.

rising input. The fact that we observe a steady-state amplified output signal for every $w_{e q}$ in Figs. 3(a) and 3(b) stems from the absence of saturation. Then, according to Eq. (10), the population difference remains unchanged. This is proved by direct computation of $w(t)$ shown in the inset of Fig. 3(a): the population difference keeps its initial value $w_{e q}=-1$ for all times.

When saturation effects are not negligible, the output signal is not stationary despite the same strong amplification of the input signal $\Omega_{0}=10^{-2} \gamma_{2}$ as before [see Figs. 3(c) and 3(d)]. Reflection $R$ and transmission $T$ slowly degrade to the level of the passive structure, because the input intensity is able to change the population difference toward the saturated value $w=0$ as clearly shown in the inset of Fig. 3(c).

So, calculations taking into account saturation demonstrate the possibility of loss compensation and signal amplification in lossy multilayers including HMMs. Albeit we are limited here to normal incidence, we anticipate the similar loss compensation valid for oblique incidence as well.

\section{RESONANT ENHANCED TRANSMISSION WITH SUPPRESSED REFLECTION IN AZO-DIELECTRIC MULTILAYERS}

In spite of simultaneous amplification of reflection and transmission shown in Figs. 3(a) and 3(b), the multilayer structure remains predominantly reflective in both passive and active regimes, whereas for the practical applications (e.g., lensing) the structure should be rather transmissive. In this section, we demonstrate the suppressed reflection regime with simultaneous enhancement of transmission. This effect takes place within a narrow frequency band; hence it is resonant by its nature.

We consider this regime in the structure with the same parameters as in the previous section, except those of the dielectric slabs (we take $d_{d}=0.12 \mu \mathrm{m}$ and $n_{d}=3.4$ ). Index $n_{d}$ is changed in order to take into account the dispersion of the semiconductor material [46], when the wavelength is within the conventional telecommunications band in the vicinity of $1.55 \mu \mathrm{m}$.

First, using the transfer-matrix method, we calculate reflection $R$ and transmission $T$ spectra of the structure with the slab permittivities given by Eqs. (1) and (12). The ratio $T / R$ in Fig. 4(a) clearly shows that reflection dominates in passive structures. In the active system, transmission takes over at the resonant wavelengths as demonstrated in Fig. 4(b) for $w_{e q}=$ -0.2. Comparative analysis of Figs. 4(a) and 4(b) reveals the same number of peaks. This allows us to conclude that the strong resonances are the amplified seed peaks available in the gainless case. The seed peaks apparently stem from the properties of the multilayer. If one substitutes the multilayer with a homogeneous anisotropic material, the permittivity of which is given by Eq. (14), the resonances move to other wavelengths due to the inaccuracies inherent in the effective medium approximation.

In the case of lossless oxide the number of amplified peaks increases, but their intensity noticeably drops [compare dashed blue lines in Fig. 4(c) with the spectrum in Fig. 4(b), where the peak of the $T / R$ ratio is up to 70]. Therefore, the conducting properties are crucial for the enhanced transmission, since the loss results in redistributing energy to the few narrower resonances. When the system is fully dielectrical, i.e., $\varepsilon_{m}$ is not negative, the resonances providing the amplified transmission at the expense of the reflection exist up to long wavelengths [Fig. 4(c)]. In the frequency domain, it is clearly observed that the peaks are separated by the same interval and correspond to the Fabry-Pérot resonances between the boundaries of the multilayer.

We take wavelength $\lambda=1.555 \mu \mathrm{m}$ of the incident wave corresponding to the transmission peak [see Fig. 4(b), where this resonance is marked with the arrow]. The transfer-matrix method estimates decrease in reflection from 0.38 to 0.07 and increase in transmission from 0.05 to 0.75 as pumping parameter $-w_{e q}$ grows from 0 to 0.2 . Since the spectra in Fig. 4 provide only a qualitative stationary picture, we simulate propagation taking into account nonuniformity of the light field across the layers and saturation of the gain medium response. To suppress the effect of saturation, the input wave amplitude is chosen to be $\Omega_{0}=10^{-4} \gamma_{2}$. After establishment of the stationary regime, the passive structure reflects about 

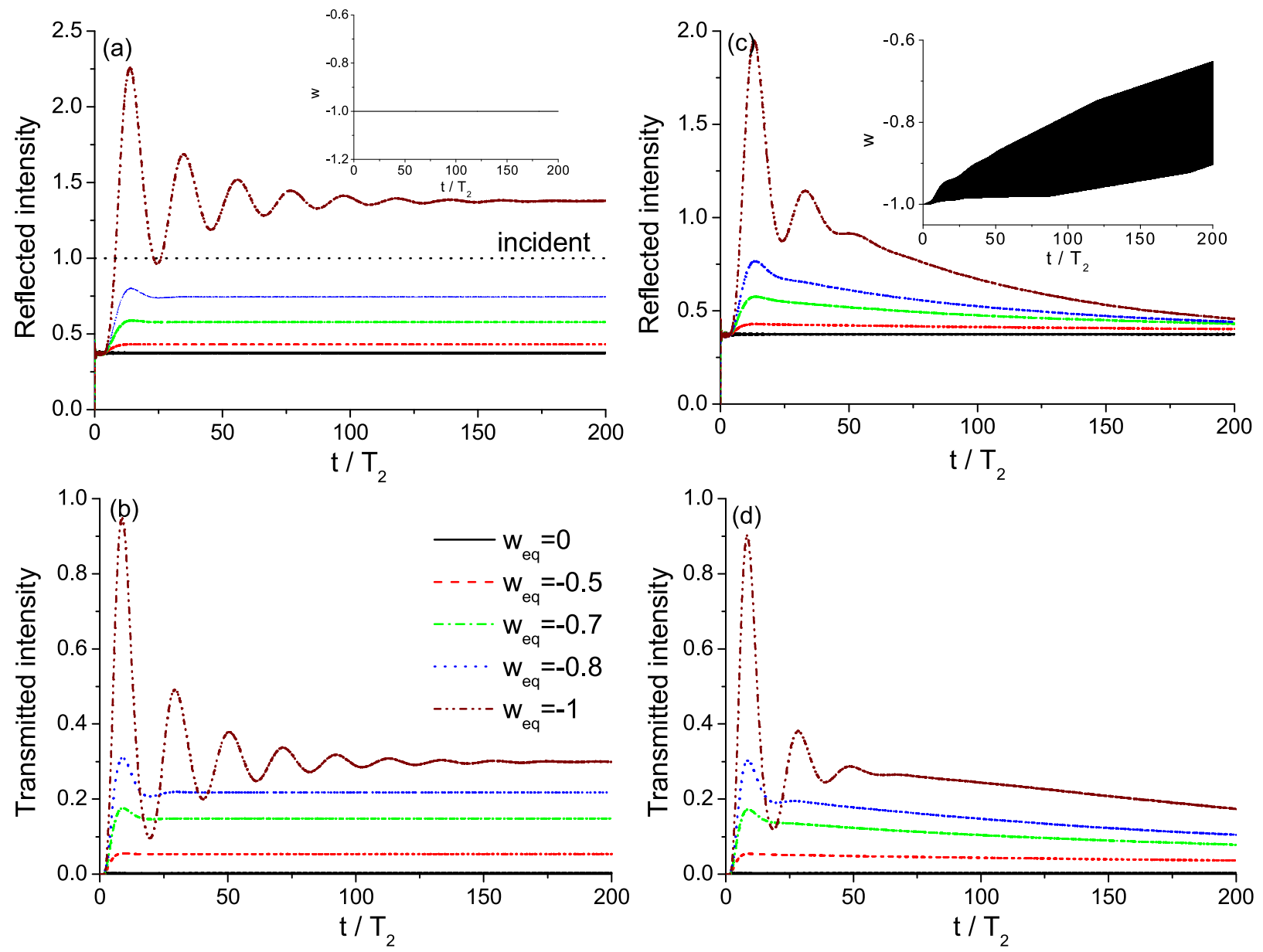

FIG. 3. Temporal dynamics of (a),(c) reflected and (b),(d) transmitted intensities for different $w_{\text {eq }}$ provided the cw input has the amplitude (a),(b) $\Omega_{0}=10^{-4} \gamma_{2}$ and (c),(d) $\Omega_{0}=10^{-2} \gamma_{2}$. Dynamics of the population difference at the entrance of the first gain layer for each of two input amplitudes $\Omega_{0}$ and pumping parameter $w_{e q}=-1$ are shown in the insets of (a) and (c). Wavelength equals $\lambda=2.5 \mu \mathrm{m}$; other parameters are the same as in Fig. 2.

$40 \%$ of incident light and transmits almost nothing (Fig. 5). This is in full accordance with the transfer-matrix calculations. For the greater $-w_{e q}$, the establishment of the stationary regime takes more time, reflection gradually decreases, and, on the contrary, transmission increases. They are approximately equal at $w_{e q}=-0.13$ as demonstrated by the corresponding curves in Fig. 5. The reflection coefficient reaches its minimal stationary value at $w_{e q}=-0.18$ with the transmission larger than $45 \%$ [Fig. 5(b)]. Higher pumping leads to the rapid growth of both reflection and transmission coefficients. At $w_{e q} \approx-0.3$, the system switches to another operation mode, in which the stored pumped energy is promptly released in the
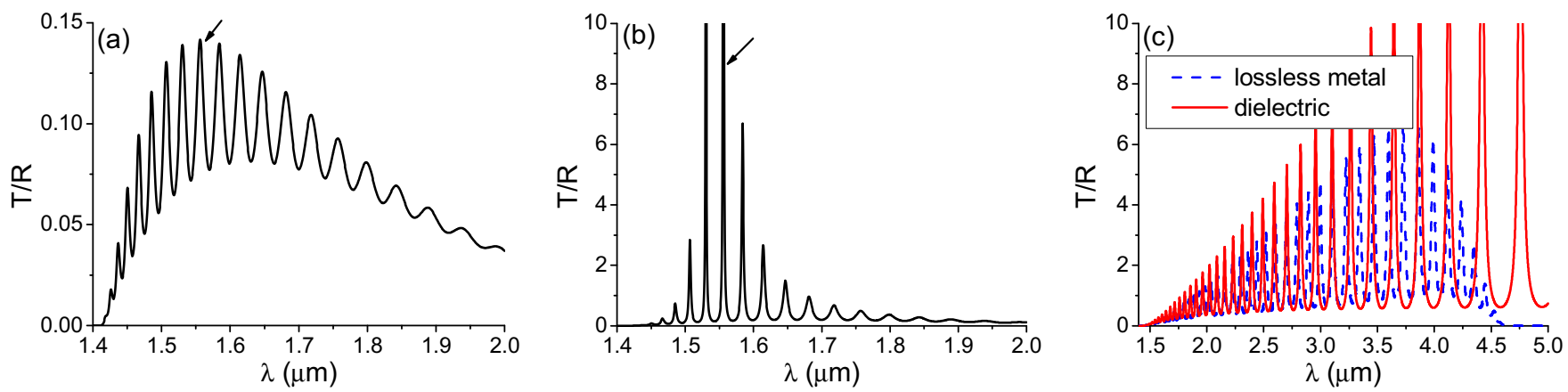

FIG. 4. Ratio of the transmission to reflection spectra of the AZO-dielectric structure for the pumping parameters (a) $w_{e q}=0$ and (b) $w_{e q}=-0.2$. (c) The ratio $T / R$ in the case of lossless $(\gamma=0)$ oxide-dielectric and entirely dielectric $\left(\varepsilon_{m}=3.45\right)$ systems (the corresponding curves are dashed blue and solid red) for the pumping parameter $w_{e q}=-0.2$. Thicknesses $d_{m}=0.1 \mu \mathrm{m}$ and $d_{d}=0.12 \mu \mathrm{m}$ and refractive index $n_{d}=3.4$, while the other parameters of calculations are the same as in the caption of Fig. 2. All calculations are carried out using the transfer-matrix method. 

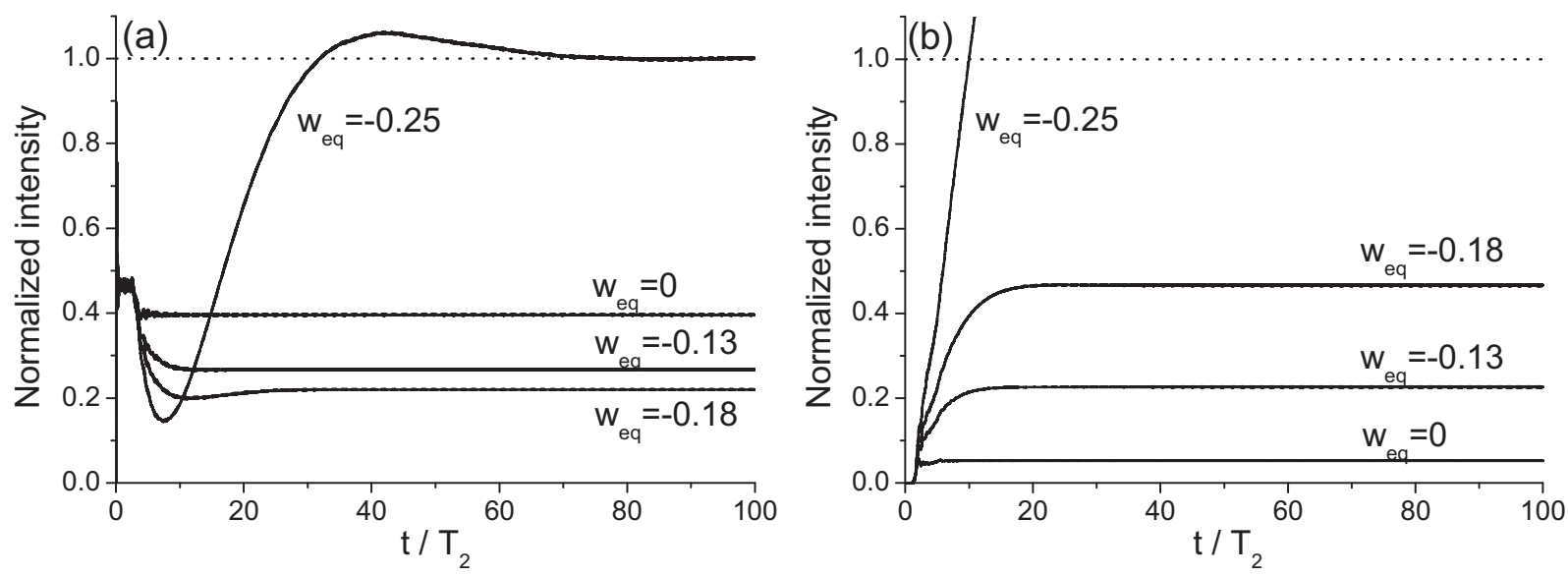

FIG. 5. Temporal dynamics of (a) reflected and (b) transmitted intensity for different pumping parameters. Wavelength $\lambda=1.555 \mu \mathrm{m}$ (C-telecom band) corresponds to the peak marked with the arrow in Fig. 4(b). Parameters of calculations can be found in the caption of Fig. 4.

form of a powerful pulse. This regime, that can be called the lasinglike mode, will be addressed afterwards.

The stationary levels of reflection $R$ and transmission $T$ depending on pumping parameter $w_{e q}$ are depicted in Fig. 6(a). There is a good agreement between the results obtained from direct numerical simulations (symbols) and transfer-matrix calculations (lines). Intersection of the reflection and transmission curves at $w_{e q} \approx-0.13$ separates two regimes (reflective and transmissive) of the system. The optimal conditions for reflecting and transmitting states are realized for small pumping parameters and for $w_{e q} \approx-0.2$, respectively. There is a mixture of the states near the crossing point of the reflection and transmission curves, and the transition of the structure from the reflective regime to the transmissive one occurs. It should be noted that the stationary transfer-matrix calculations not only specify the perspective frequency band containing the resonance, but also correctly predict the intersection point and optimal pumping for minimal reflection. On the other hand, this method cannot describe peculiarities of the transition between two states and generally overestimates the depth of the reflection minimum as clearly shown in Fig. 6(a). We should note that almost identical dependencies as shown in this figure can be obtained for the total reflection and transmission energies of incident pulses with durations long enough to be considered as quasimonochromatic. Figure 6(b) shows the transfer-matrix results for the stationary level of reflection and transmission in the full range of pumping parameters $w_{e q}$. The peaks in the vicinity of $w_{e q} \approx-0.3$ can be treated as a qualitative indication of the lasinglike mode, though the quantitative characteristics of this regime can be revealed only by solving the full system of equations described in Sec. II. The optimal pumping parameter for the transmissive state of the system extracted from the transmission-to-reflection ratio [solid line in Fig. 6(c)] well correlates with the numerical simulations (symbols), but the value of this ratio is strongly overestimated by the transfer-matrix method. This can be explained by the detuning of the resonant frequency in the FDTD approach as compared with the transfer-matrix method.

For relatively low pumping parameters $-w_{e q}<0.28$ as in Fig. 6(a) there is no saturation, and population difference $w$ in the gain medium does not vary with time. Even at the edge value $w_{e q}=-0.3$ the population difference is time independent, while the reflected and transmitted signals in Fig. 7(a) demonstrate slowly decaying oscillations, i.e., there is a very long transient period in this case. Therefore, we cannot assign any steady-state value to reflection and transmission for the intermediate parameter range $-0.35 \lesssim w_{e q} \lesssim-0.28$. Nevertheless, when the pumping crosses over a certain threshold level, saturation comes into play. The temporal dependence of the population difference starts as an accrescent fluctuation analogous to that shown in the inset of Fig. 3(c), while the amplified signal takes the form of a pulse with the peak intensity exceeding the input cw intensity in hundreds or even thousands times. The example for $w_{e q}=-0.4$ shown in Fig. 7(b) demonstrates almost identical pulses in both directions. The pulse duration shortens for higher pumping. This phenomenon of powerful pulse generation differs from the usual lasing action, because the response develops not from fluctuations of the light field inside the structure, but rather from the strong external radiation. This is the reason why we call this regime the lasinglike mode. We should also emphasize that the transition to this mode has the resonant character (compare with Fig. 3 where lasinglike regime is absent in the full range of pumping) and has approximately the same threshold for resonances in different frequency ranges.

Pulsed-form amplification is followed by formation of the stationary response after the pumped energy has been emitted. At $w_{e q}=-0.4$, transmission is higher than reflection, they are almost equal at $w_{e q}=-0.5$, and then reflection (transmission) increases (decreases) keeping absorption level approximately at the same level of 0.2 [see symbols in Fig. 7(c)]. The structure becomes almost entirely reflective in the large-time limit at $w_{e q}=-1$. We should emphasize that the system does not return to the gainless state, since reflection is much higher than that found in the passive system. Note that the transfermatrix calculations give strongly overestimated values of the stationary level of reflection [see lines in Fig. 7(c)], because the inhomogeneous field distribution and saturation within the gain layers cannot be ignored in this case.

\section{RESONANT REFLECTIONLESS TRANSMISSION FOR OBLIQUELY INCIDENT AND EVANESCENT WAVES}

An obliquely incident wave is characterized by transverse $k_{x}$ and longitudinal $k_{z}$ wave numbers directed along and across 

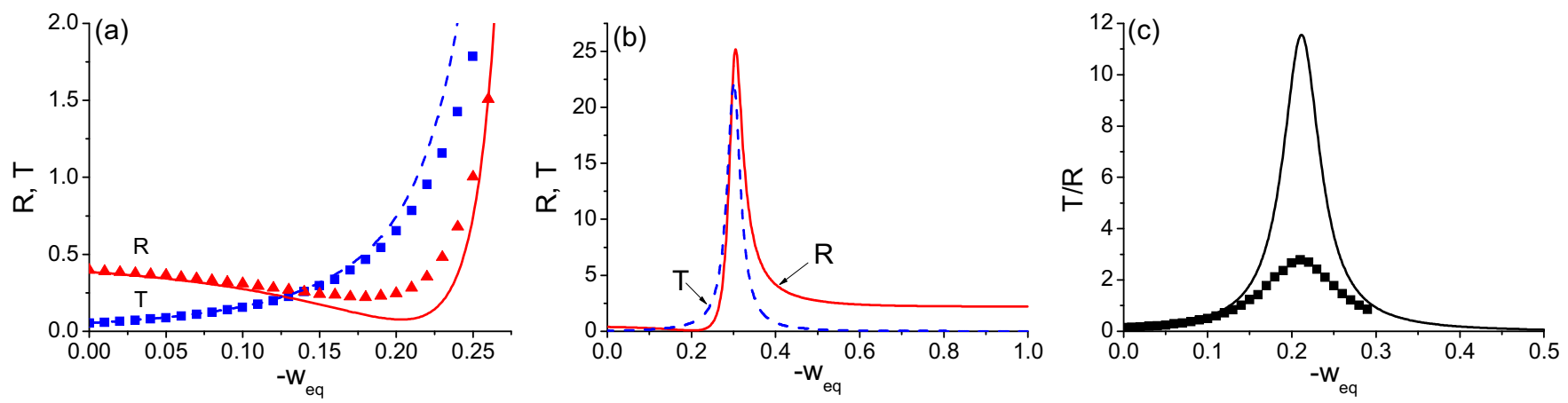

FIG. 6. Dependence of the stationary level of reflection (R) and transmission (T) on the pumping parameter $w_{e q}$ at $\lambda=1.555 \mu \mathrm{m}$. (a) Results of numerical simulations (symbols) and transfer-matrix calculations (lines). (b) Transfer-matrix results in the full range of pumping parameter. (c) Transmission to reflection ratio from the numerical simulations (symbols) and transfer-matrix calculations (line).

the layers, respectively. Transverse component is conserved at the multilayer interfaces being defined by incident angle $\alpha$ of the input wave as follows: $k_{x}=k_{0} \sin \alpha$ (the ambient medium is free space). If an evanescent wave is excited at the input, then transverse wave number $k_{x}>k_{0}$, while longitudinal wave number $k_{z}$ is defined by the corresponding dispersion equation.

Transmission of the obliquely incident and evanescent waves is sensitive to their polarization, which can be easily noticed within the effective medium approximation [see Eqs. (13) and (14)]. TE-polarized plane waves in such a homogenized medium are the ordinary waves of the effective uniaxial crystal with the $z$-oriented optical axis. Their dispersion is governed by the equation for isotropic materials: $k_{x}^{2}+k_{z}^{2}=$ $\varepsilon_{x x} k_{0}^{2}$. Since $\operatorname{Re}\left(\varepsilon_{x x}\right)>0$ (see Fig. 2), propagation of waves with longitudinal wave numbers $k_{x}$ up to $k_{0} \sqrt{\operatorname{Re}\left(\varepsilon_{x x}\right)}$ is allowed. Dispersion equation of TM-polarized (extraordinary) plane waves reads $k_{x}^{2} / \varepsilon_{z z}+k_{z}^{2} / \varepsilon_{x x}=k_{0}^{2}$. If $\operatorname{Re}\left(\varepsilon_{z z}\right)>0$, the tangential wave number cannot be greater than $k_{0} \sqrt{\operatorname{Re}\left(\varepsilon_{z z}\right)}$. Otherwise, when $\operatorname{Re}\left(\varepsilon_{z z}\right)<0$, the structure can, in principle, sustain an evanescent wave with any $k_{x}$.

In this section, the calculations are carried out using the transfer-matrix method. According to Fig. 8, the peaks of active and passive structures agree with each other for the evanescent waves. The system exhibits resonant amplification for each pumping $w_{e q}$, transmission maximums being generally stronger than reflection ones. Out of the resonances the system is strongly absorbing [see Fig. 8(c)]. Points of low reflection result in the maximums of $T / R$. Figure 9 clearly demonstrates that the evanescent waves can be amplified exclusively in transmission. Indeed, similar to the case of normal incidence (Fig. 4), ratio $T / R$ is small for the passive structure and strongly enhanced for active multilayers [see Fig. 9(a)]. The greater is the pumping parameter, the larger is the redshift of the major peak. Efficiency of amplification apparently depends not only on $w_{e q}$, but also on the spectral characteristics of the passive structure. In order to study the features of the waves at the peaks of $T / R$, we find transverse wave numbers $k_{x}$ of the waves corresponding to one of such peaks as a function of wavelength $\lambda$. In other words, there is a correspondence between the position of the peak $\lambda$ and wave number $k_{x}$ which provides the maximum of the $T / R$ ratio. The procedure of retrieving dispersion $k_{x}(\lambda)$ is as follows. Transmission and reflection depend on $\lambda$ and $k_{x}$. For each wavelength $\lambda$, we introduce wave numbers $k_{x}^{(j)}(j=1, \ldots, M)$ in the chosen region, calculate $T\left(\lambda, k_{x}^{(j)}\right) / R\left(\lambda, k_{x}^{(j)}\right)$, and determine the wave number corresponding to the maximal ratio. Thus we have the pair $\left(\lambda, k_{x}\right)$. The whole set of such pairs results in the dispersion curve $k_{x}(\lambda)$. Then we calculate transmission $T$ and reflection $R$ coefficients for the values lying on the dispersion curve. A slight jitter of the values in Fig. 9(b) appears due to inaccuracy of finding the peak values of the narrow resonances $T / R$. The strongest jitter occurs near $k_{z}=0$ [wavelength is about $1.6 \mu \mathrm{m}$ in Fig. 9(b)]. To find the correct
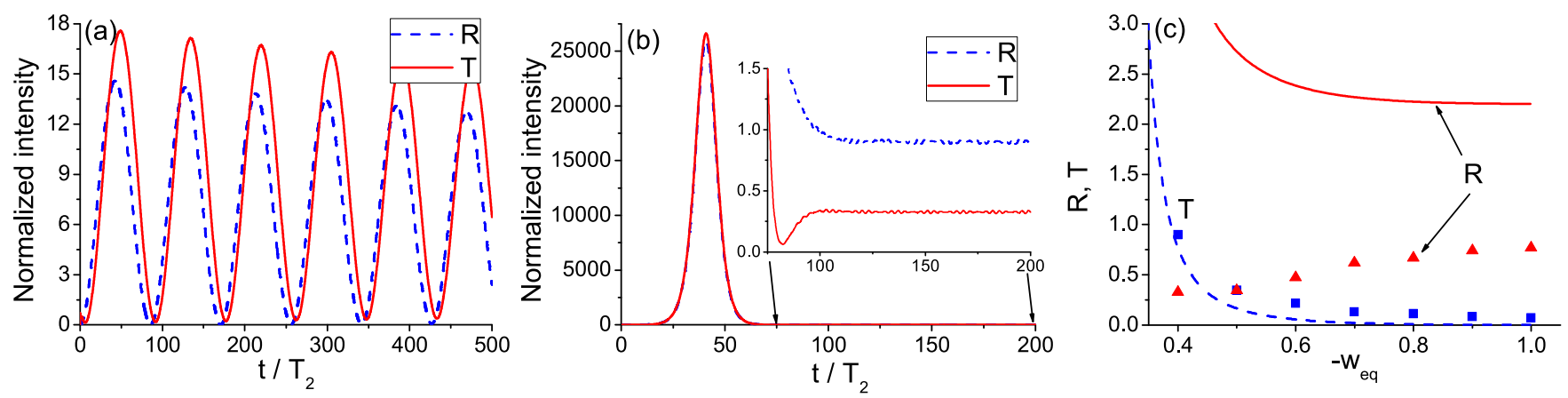

FIG. 7. (a) Temporal dynamics of the reflected (R) and transmitted (T) intensity for the pumping parameter $w_{e q}=-0.3$. (b) The same as in (a), but for $w_{e q}=-0.4$. (c) Dependence of the stationary level of reflection (R) and transmission (T) on the pumping parameter for the structure in the lasinglike mode derived from the numerical simulations (symbols) and transfer-matrix calculations (lines). Parameters of calculations are the same as in the caption of Fig. 4. 

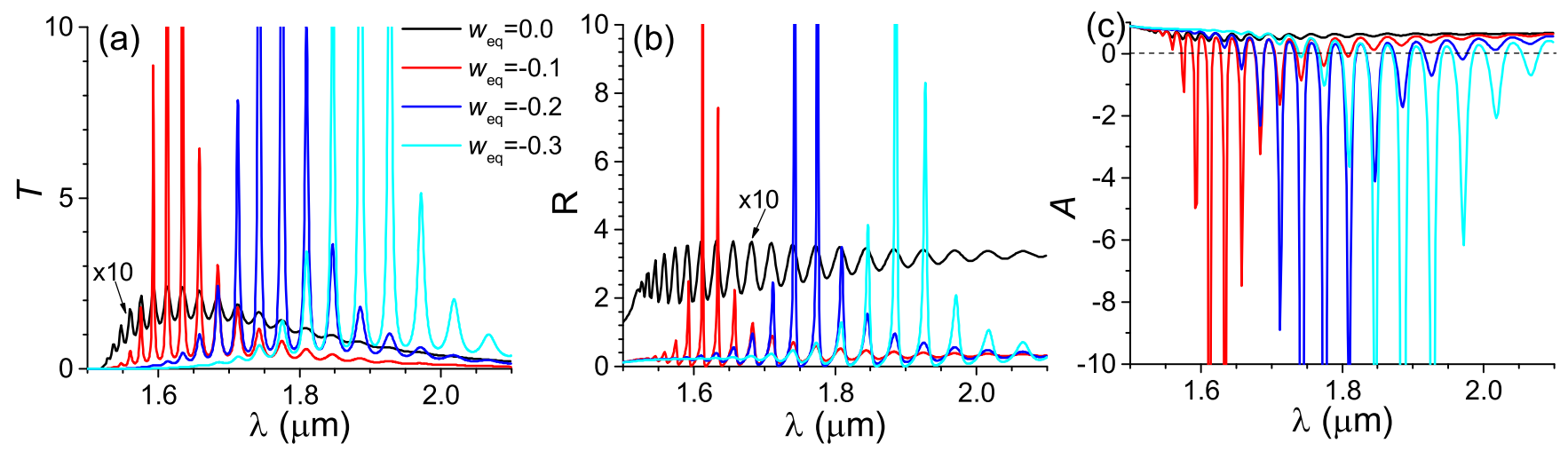

FIG. 8. (a) Transmission $T$, (b) reflection $R$, and (c) absorption $A=1-T-R$ for the TE-polarized evanescent wave $\left(k_{x}=2 k_{0}\right)$ propagating through the passive and active multilayers $\left(d_{m}=100 \mathrm{~nm}\right.$ and $\left.d_{d}=200 \mathrm{~nm}\right)$. Parameters of calculations are the same as in Fig. 2.

positions of the narrow peaks in this region and, therefore, suppress the deviations, we increase the accuracy of our calculation (number $M$ of discrete wave numbers) in 100 times there.

When $k_{x}(\lambda)<k_{0}$, we are in the realm of propagating waves. In this regime, reflection completely vanishes and transmission is unity for a single obliquely incident wave with $k_{x} \approx 0.75 k_{0}$ (the angle of incidence is $\alpha_{0}=48.5^{\circ}$ ), which corresponds to the wavelength of the peak around $1.98 \mu \mathrm{m}$ [see the vertical dotted line in Fig. 9(b)]. Both $T$ and $R$ rapidly grow for angles $\alpha>\alpha_{0}$. Enormous values of transmission and reflection resemble those observed in Fig. 6(b). The transfermatrix method is likely to be inapplicable for quantitative conclusions, but it should work quite well in the regime of high transmission with suppressed reflection. If $\alpha<\alpha_{0}$, transmission drops and becomes equal to reflection near $k_{x}=0$ [see the far right part of Fig. 9(b)]. It is interesting that the incident evanescent waves $\left(k_{x}>k_{0}\right)$ can result in higher $T / R$ than the propagating waves. Moreover, the regime of high transmission with well suppressed reflection is observed
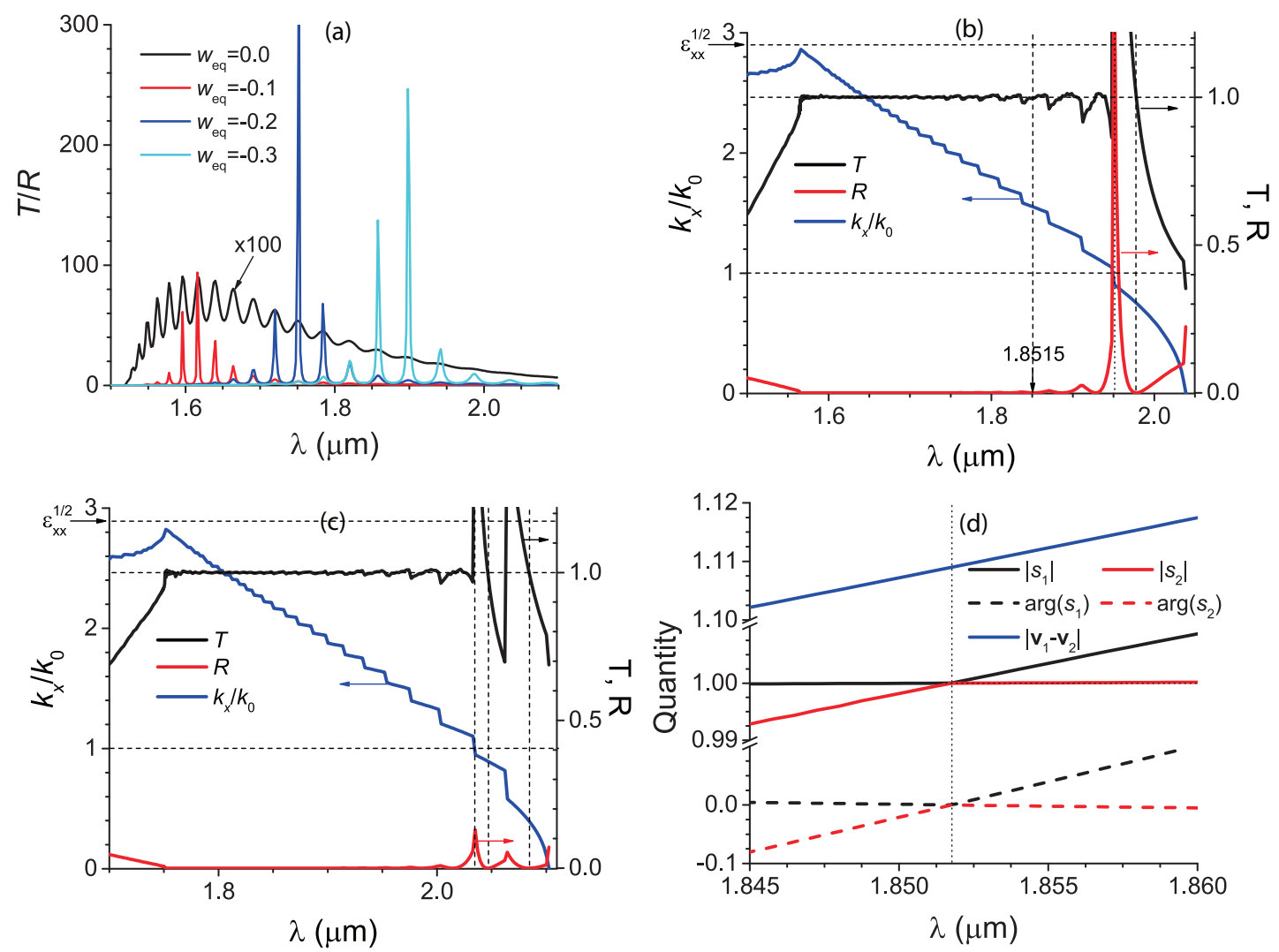

FIG. 9. (a) Transmission [Fig. 8(a)] to reflection [Fig. 8(b)] ratio. Dispersion equation $k_{x}(\lambda)$ of the peak value of the ratio $T / R$ (left scale) and transmission $T$ and reflection $R$ spectra at this peak (right scale) for TE-polarized waves in the active multilayers $\left(d_{m}=100 \mathrm{~nm}\right.$ and $d_{d}=200 \mathrm{~nm}$ ): (b) $w_{e q}=-0.2$ and (c) $w_{e q}=-0.3$. (d) Eigenvalues $s_{1}$ and $s_{2}$ and difference of eigenvectors $\left|\mathbf{v}_{1}-\mathbf{v}_{2}\right|$ of scattering matrix near the point of $T=1$ and $R=0[\lambda \approx 1.8515$ in (b)]. Other parameters of calculations are the same as in Fig. 2. 
in the wide spectral range and ends at $k_{x}=k_{0} \sqrt{\operatorname{Re} \varepsilon_{x x}}$. However, full transmission with no reflection exists only at the specific wavelengths. When pumping parameter $w_{e q}$ decreases (increases), the picture in Fig. 9(b) red (blue) shifts, because the higher gain is able to compensate the higher multilayer losses shown in Fig. 2(b). Behavior of the system at oblique incidence $\left(k_{x}<k_{0}\right)$ may also change, e.g., revealing several wavelengths of full transmission as demonstrated in Fig. 9(c). When the thicknesses of the AZO and dielectric slabs are enlarged, we approach the photonic crystal mode, and the moderate oscillations observed in transmission of the evanescent waves in Figs. 9(b) and 9(c) become more pronounced.

To achieve reflectionless transmission, we do not impose any specific condition on the system in question, although the full loss compensation can be forbidden in the quasistatic consideration of plasmonic structures [51]. On the other hand, $\mathcal{P} \mathcal{T}$-symmetric loss-gain systems demonstrate transmission without reflection [52].

Let us look closer at the behavior of the system near the points of full transmission. For instance, both conditions $T=1$ and $R=0$ are met in the vicinity of wavelength $\lambda=$ $1.8515 \mu \mathrm{m}$ [see Fig. 9(b)]. Since reflectionless transmission is known for $\mathcal{P} \mathcal{T}$-symmetric structures, it is instructive to compare them with the system under consideration. We calculate the scattering matrix $S$ and similar to $\mathcal{P} \mathcal{T}$-symmetric structures [31], we obtain $s_{1}=s_{2}=1$ at the point characterized by $T=1$ and $R=0$ [see the absolute values and arguments for complex eigenvalues in Fig. 9(d)]. However, our system only resembles the $\mathcal{P} \mathcal{T}$-symmetric one at the exceptional point, because, first, it does not have the necessary distribution of permittivity $\varepsilon(z)=\varepsilon^{*}(-z)$ and, second, the eigenvectors of the scattering matrix are not the same at this point [see their difference in Fig. 9(d)]. Since the system is not $\mathcal{P} \mathcal{T}$ symmetric, the condition of symmetry-broken state $\left|s_{2}\right|=1 /\left|s_{1}\right|$ is not valid, too. We believe that the emergence of the points of reflectionless transmission is caused by resonant properties of the multilayers.

As demonstrated in Fig. 2, there is a spectral range of hyperbolic dispersion from $2.2 \mu \mathrm{m}$ to $3.1 \mu \mathrm{m}$ available for the TM-polarized waves. However, the resonant reflectionless transmission is not observed for these waves. The suppressed reflection in this case is caused by the Brewster effect as shown in Fig. 10 [compare the curve for the Brewster effect in the effective medium approximation with curve $k_{x}(\lambda)$ ]. Evanescent waves possess a much lower $T / R$ ratio compared to the propagating waves and, hence, are absent in the dispersion curve of Fig. 10.

\section{CONCLUSION}

The system of alternating transparent conducting oxide (AZO) and amplifying dielectric (semiconductor doped with quantum dots) slabs has been studied in two modes: loss compensation and resonant low-reflection transmission. Using the FDTD method, we have shown that the stationary regime is rapidly established giving us the opportunity to exploit the transfer-matrix approach for the realistic description of the system. In the loss compensation mode, we have analyzed temporal evolution of the reflected and transmitted signals

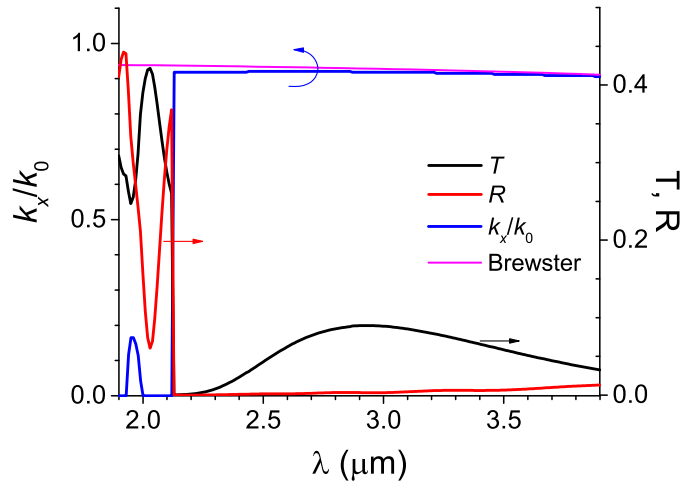

FIG. 10. Dispersion equation $k_{x}(\lambda)$ of the peak value of the ratio $T / R$ (left scale) and transmission $T$ and reflection $R$ spectra at this peak (right scale) for TM-polarized waves in the active metaldielectric multilayer with $d_{m}=20 \mathrm{~nm}, d_{d}=40 \mathrm{~nm}$, and $w_{e q}=-0.2$. Other parameters of calculations are the same as in the caption of Fig. 2.

with and without saturation demonstrating that saturation suppresses signal amplification in the long-time limit. Harnessing the resonant properties of multilayers, we have found the conditions for transmission enhancement with simultaneous suppression of reflection. The resonant wavelengths can be estimated within the transfer-matrix approach for stationary fields. Temporal dynamics qualitatively confirms existence of the maximum in the ratio of transmission to reflection, but the specific value of $T / R$ differs. Since the resonance is narrow, this difference is likely caused by a small deviation of the resonant frequency in FDTD and transfer-matrix calculations. We have revealed the lasinglike mode at large enough pumping, the appearance of which is again associated with the multilayer resonances. In this mode, both transmitted and reflected fields are released as powerful pulses with subsequent formation of the stationary levels of reflection and transmission, which cannot be predicted correctly by the transfer-matrix approach.

Interaction of the obliquely incident and evanescent waves with the multilayer has been studied using the transfer-matrix method. We have found a number of discrete points, where the reflection equals zero, while the transmission is unity. Although the system at these points may resemble a $\mathcal{P} \mathcal{T}$ symmetric one (the eigenvalues of the scattering matrix are equal), it is not the case, because the permittivity condition $\varepsilon(z)=\varepsilon^{*}(-z)$ is not satisfied and the eigenvectors of the scattering matrix are not the same. We guess that emergence of reflectionless transmission stems rather from the resonant properties of the multilayer, in which the Bloch waves can propagate without amplification or attenuation. It should be noted that the $\mathcal{P} \mathcal{T}$-symmetric-like behavior has been also observed in dissimilar directional couplers [53]. In our paper, the reflectionless transmission has been found only for TE-polarized obliquely incident and evanescent waves, but this may not be the rule. To conclude, we have revealed the mechanism of total transmission of evanescent waves by means of their conversion into propagating modes of the multilayer. Reflectionless transmission for evanescent waves opens new opportunities for the light-matter interaction in plasmonics and metamaterials. 


\section{ACKNOWLEDGMENTS}

This work was supported by the Belarusian Republican Foundation for Fundamental Research (Projects No. F16K016 and No. F16R-049) and the State Fund for Fundamental
Research of Ukraine (Project No. F73). Numerical simulations of light interaction with resonant media were supported by the Russian Science Foundation (Project No. 17-72-10098). Partial financial support from the Villum Fonden via the DarkSILD project and from the HyMeCav project is acknowledged.
[1] L. Novotny and B. Hecht, Principles of Nano-Optics (Cambridge University Press, Cambridge, UK, 2012).

[2] S. A. Meier, Plasmonics: Fundamentals and Applications (Springer, New York, 2007).

[3] Plasmonic Nanoguides and Circuits, edited by S. Bozhevolnyi (Pan Stanford Publishing, Singapore, 2009).

[4] A. Poddubny, I. Iorsh, P. Belov, and Yu. Kivshar, Nat. Photon. 7, 948 (2013).

[5] V.P. Drachev, V. A. Podolskiy, and A. V. Kildishev, Opt. Express 21, 15048 (2013).

[6] D. Lu and Z. Liu, Nat. Commun. 3, 1205 (2012).

[7] L. Ferrari, C. Wu, D. Lepage, X. Zhang, and Z. Liu, Prog. Quantum Electron. 40, 1 (2015).

[8] C. L. Cortes, W. Newman, S. Molesky, and Z. Jacob, J. Opt. 14, 063001 (2012).

[9] G. V. Naik, J. Kim, and A. Boltasseva, Opt. Mater. Express 1, 1090 (2011).

[10] G. V. Naik, V. M. Shalaev, and A. Boltasseva, Adv. Mater. 25, 3264 (2013).

[11] V. E. Babicheva, A. Boltasseva, and A. V. Lavrinenko, Nanophotonics 4, 165 (2015).

[12] P. Guo, R. D. Schaller, J. B. Ketterson, and R. P. H. Chang, Nat. Photon. 10, 267 (2016).

[13] Y. Wang, A. C. Overvig, S. Shrestha, R. Zhang, R. Wang, N. Yu, and L. Dal Negro, Opt. Mater. Express 7, 2727 (2017).

[14] L. Pavesi, L. Dal Negro, C. Mazzoleni, G. Franzò, and F. Priolo, Nature (London) 408, 440 (2000).

[15] H. Y. Jeong, U. J. Kim, H. Kim, G. H. Han, H. Lee, M. S. Kim, Y. Jin, T. H. Ly, S. Y. Lee, Y.-G. Roh, W.-J. Joo, S. W. Hwang, Y. Park, and Y. H. Lee, ACS Nano 10, 8192 (2016).

[16] Active Plasmonics and Tuneable Plasmonic Metamaterials, edited by A. V. Zayats and S. Maier (Wiley, Hoboken, NJ, 2013).

[17] R. F. Oulton, V. J. Sorger, T. Zentgraf, R.-M. Ma, C. Gladden, L. Dai, G. Bartal, and X. Zhang, Nature (London) 461, 629 (2009).

[18] D. A. Svintsov, A. V. Arsenin, and D. Yu. Fedyanin, Opt. Express 23, 19358 (2015).

[19] J. Zhang, H. Jiang, B. Gralak, S. Enoch, G. Tayeb, and M. Lequime, Eur. Phys. J.: Appl. Phys. 46, 32603 (2009).

[20] S. Xiao, V. P. Drachev, A. V. Kildishev, X. Ni, U. K. Chettiar, H.-K. Yuan, and V. M. Shalaev, Nature (London) 466, 735 (2010).

[21] X. Ni, S. Ishii, M. D. Thoreson, V. M. Shalaev, S. Han, S. Lee, and A. V. Kildishev, Opt. Express 19, 25242 (2011).

[22] R. S. Savelev, I. V. Shadrivov, P. A. Belov, N. N. Rosanov, S. V. Fedorov, A. A. Sukhorukov, and Yu. S. Kivshar, Phys. Rev. B 87, 115139 (2013).

[23] C. Argyropoulos, N. Mohammadi Estakhri, F. Monticone, and A. Alù, Opt. Express 21, 15037 (2013).

[24] T. Galfsky, H. N. S. Krishnamoorthy, W. Newman, E. E. Narimanov, Z. Jacob, and V. M. Menon, Optica 2, 62 (2015).

[25] V. N. Pustovit, A. M. Urbas, and D. E. Zelmon, Phys. Rev. B 94, 235445 (2016).
[26] B. Janaszek, A. Tyszka-Zawadzka, and P. Szczepański, Opt. Express 25, 13153 (2017).

[27] C. M. Bender and S. Boettcher, Phys. Rev. Lett. 80, 5243 (1998).

[28] R. El-Ganainy, K. G. Makris, D. N. Christodoulides, and Z. H. Musslimani, Opt. Lett. 32, 2632 (2007).

[29] C. E. Rüter, K. G. Makris, R. El-Ganainy, D. N. Christodoulides, M. Segev, and D. Kip, Nat. Phys. 6, 192 (2010).

[30] K. G. Makris, R. El-Ganainy, D. N. Christodoulides, and Z. H. Musslimani, Phys. Rev. Lett. 100, 103904 (2008).

[31] L. Ge, Y. D. Chong, and A. D. Stone, Phys. Rev. A 85, 023802 (2012).

[32] Z. Lin, H. Ramezani, T. Eichelkraut, T. Kottos, H. Cao, and D. N. Christodoulides, Phys. Rev. Lett. 106, 213901 (2011).

[33] Ya. V. Kartashov, C. Hang, V. V. Konotop, V. A. Vysloukh, G. Huang, and L. Torner, Laser Photon. Rev. 10, 100 (2016).

[34] S. V. Suchkov, A. A. Sukhorukov, J. Huang, S. V. Dmitriev, C. Lee, and Yu. S. Kivshar, Laser Photon. Rev. 10, 177 (2016).

[35] V. V. Konotop, J. Yang, and D. A. Zezyulin, Rev. Mod. Phys. 88, 035002 (2016).

[36] C. Fietz and C. M. Soukoulis, Opt. Express 20, 11548 (2012).

[37] F. Rüting, J. Cuerda, J. Bravo-Abad, and F. J. Garcia-Vidal, Laser Photon. Rev. 8, L65 (2014).

[38] A. Veltri, A. Chipouline, and A. Aradian, Sci. Rep. 6, 33018 (2016).

[39] V. R. Tuz, D. V. Novitsky, P. L. Mladyonov, S. L. Prosvirnin, and A. V. Novitsky, J. Opt. Soc. Am. B 31, 2095 (2014).

[40] D. V. Novitsky, Phys. Rev. A 79, 023828 (2009).

[41] D. V. Novitsky, Phys. Rev. A 84, 013817 (2011).

[42] M. E. Crenshaw, Phys. Rev. A 78, 053827 (2008).

[43] D. V. Novitsky, J. Opt. Soc. Am. B 28, 18 (2011).

[44] P. Markoš and C. M. Soukoulis, Wave Propagation: From Electrons to Photonic Crystals and Left-handed Materials (Princeton University Press, Princeton, NJ, 2008).

[45] E. Shkondin, O. Takayama, M. E. Aryaee Panah, P. Liu, P. V. Larsen, M. D. Mar, F. Jensen, and A. V. Lavrinenko, Opt. Mater. Express 7, 1606 (2017).

[46] Handbook of Optical Constants of Solids, edited by E. D. Palik (Academic Press, San Diego, 1998).

[47] J.-C. Diels and W. Rudolph, Ultrashort Laser Pulse Phenomena, 2nd ed. (Academic Press, San Diego, 2006).

[48] S. Savoia, G. Castaldi, V. Galdi, A. Alù, and N. Engheta, Phys. Rev. B 91, 115114 (2015).

[49] V. E. Babicheva, I. V. Kulkova, R. Malureanu, K. Yvind, and A. V. Lavrinenko, Photon. Nanostruct.-Fund. Appl. 10, 389 (2012).

[50] G. V. Naik, J. Liu, A. V. Kildishev, V. M. Shalaev, and A Boltasseva, Proc. Natl. Acad. Sci. USA 109, 8834 (2012).

[51] M. Stockman, Philos. Trans. R. Soc. London A 369, 3510 (2011).

[52] O. V. Shramkova and G. P. Tsironis, Phys. Rev. B 94, 035141 (2016).

[53] W. Walasik, C. Ma, and N. M. Litchinitser, New J. Phys. 19, 075002 (2017). 